\title{
Insulin Regimens to Treat Hyperglycemia in Hospitalized Patients on Nutritional Support: Systematic Review and Meta-Analyses
}

\author{
Marina Verçoza Viana ${ }^{a, b}$ Luciana Verçoza Viana ${ }^{a, c}$ Ana Laura Tavares ${ }^{a}$ \\ Mirela Jobim de Azevedo a, c \\ ${ }^{a}$ Division of Endocrinology, ${ }^{b}$ Critical Care Unit, and ${ }^{\mathrm{C}}$ Medical Nutrition Division, Hospital de Clínicas de Porto Alegre, \\ Universidade Federal do Rio Grande do Sul, Porto Alegre, Brazil
}

\section{Keywords}

Nutritional support · Hospitalized patients · Insulin ·

Glycemic control

\begin{abstract}
Background: The best insulin regimen to treat hyperglycemia in hospitalized patients on nutritional support (NS) is unclear. Methods: We searched electronic databases to identify cohort studies or randomized clinical trials in order to evaluate the efficacy of different insulin regimens used to treat hyperglycemia in hospitalized patients on NS on diverse outcomes: mean blood glucose (MBG), hypoglycemia, length of stay in hospital, and mortality. Results: Seventeen studies from a total of 5,030 were included. Enteral Group included 8 studies; 1,203 patients using rapid, glargine, NPH, or Premix insulin; MBG 108-225 mg/dL; hypoglycemia 0-13\%. In indirect meta-analyses, NPH insulin ranked best for glucose control (MD 95\% Cl $-2.50 \mathrm{mg} / \mathrm{dL}$ [2.65 to -2.35$]$ ). Parenteral Group included 4 studies; 228 patients using regular and glargine or NPH insulin; MBG 137-202 mg/dL; hypoglycemia 0-40\%. In meta-analyses comparing regular insulin added to parenteral nutrition bag with glargine, MBG (MD $95 \% \mathrm{Cl}-3.78 \mathrm{mg} / \mathrm{dL}$ [-11.93 to 4.37$\left.] ; P^{2}=0 \%\right)$ or hypoglyce-
\end{abstract}

\section{KARGER}

(C) 2017 S. Karger AG, Basel

E-Mail karger@karger.com

www.karger.com/anm mia frequency (RR 95\% Cl 1.37 [0.43-4.32]; $\left.\right|^{2}=70.7 \%$ ) did not differ. The description related to hospital length of stay and mortality was inconsistent between groups. Conclusions: The best insulin regimen to treat hyperglycemia in hospitalized patients on NS has not been established; best results using insulin regimens with NPH in enteral nutrition do not seem to be clinically relevant.

(c) 2017 S. Karger AG, Basel

\section{Introduction}

Hyperglycemia is a costly and common health care problem in hospitalized patients with and without diabetes. The risk of hospital complications increases with illness severity and also in patients without a previous history of diabetes [1]. The mechanism underlying hyperglycemia during acute illness is complex and includes the release of counter-regulatory hormones (corticosteroids and catecholamines) and proinflammatory mediators and administration of exogenous corticosteroids, vasopressors, and enteral and parenteral nutrition [2].

The objective of nutritional support (NS), including both enteral and parenteral nutrition, is to prevent the ef-

Marina Verçoza Viana

Division of Endocrinology, Hospital de Clínicas de Porto Alegre Rua Ramiro Barcelos 2350

Porto Alegre, RS 90035-003 (Brazil)

E-Mail marivv1981@gmail.com 
fects of starvation, such as increased risk of death and/or infection, and occasionally to alter favorably the natural course or treatment of a specific disease [3, 4]. Enteral nutrition, whenever possible, is preferred over parenteral nutrition because there are less associated complications, including hyperglycemia and costs [5].

The Van den Berghe et al. [6] seminal randomized clinical trial (RCT) conducted in critically ill surgical patients demonstrated that treatment of hyperglycemia by continuous intravenous insulin administration attaining strict glycemic control (80-110 mg/dL) reduced length of stay in morbidity intensive care unit (ICU) by 5 days, and surgical mortality in critically ill patients. Since then, the treatment of hyperglycemia in hospitalized patients has become an important target of clinical care. However, these results were not confirmed in the NICE-SUGAR multicenter study, where increased mortality occurred for ICU patients on strict glucose control using intensive insulin therapy and no reduction on length of stay in hospital was observed [7]. Subsequently, different strategies to reach glycemic control have been proposed for hospitalized patients inside and outside the ICU.

In general, up to $30 \%$ of hospitalized patients received some type of specialized NS [8]. Usually, a high frequency of hyperglycemia is observed in these patients. Depending on the definition of hyperglycemia, its prevalence varies from 44 to $90 \%$ [9] for patients receiving parenteral nutrition and around $40 \%$ [10] for patients on enteral nutrition. Clinical societies have different thresholds for hyperglycemia $[11,12]$. According to the Americand Diabetes Association, insulin therapy should be initiated for treatment of persistent hyperglycemia starting threshold $180 \mathrm{mg} / \mathrm{dL}$. Once insulin therapy is started, a target glucose range 140$180 \mathrm{mg} / \mathrm{dL}$ is recommended for the majority of critically and noncritically ill patients [12]. However, even considering the great number of patients who need to treat hyperglycemia and its associated complications in daily clinical practice, there are no guidelines to recommend the best regimen of insulin therapy to treat hyperglycemia in NS.

The aim of this study was to evaluate the effect of different insulin regimens used to treat hyperglycemia in hospitalized patients receiving NS - enteral, parenteral, or both - on pre-established outcomes: hyperglycemia, hypoglycemia, hospital length of stay, and mortality.

\section{Methods}

This systematic review was carried out using a protocol constructed according to the Cochrane Handbook recommendations [13] and reported in accordance with Preferred Reporting Items for Systematic Reviews and Meta-Analyses statement [14]. The study protocol was registered in the PROSPERO International prospective register of systematic reviews (CRD42015015749).

\section{Data Sources and Searches}

We searched databases from Medline, Embase, ISI web of science, ClinicalTrials.gov register, Cochrane, and Scopus, up to March 2017, to identify studies that compared different insulin regimens in hospitalized patients receiving either enteral or parenteral NS. In addition, a manual search was performed in the reference lists of included articles. We contacted the authors by mail if essential data were not clearly described in a study.

The initial search strategy was defined by the following MESH terms: "Nutritional support"[Mesh] or "Enteral nutrition"[Mesh] or "Parenteral nutrition, Total" [Mesh] or "Parenteral nutrition, Solutions"[Mesh] combined with "Insulin"[Mesh] or "Insulin lente"[Mesh] or "Insulin ultralente"[Mesh] or "Insulin regular, human"[Mesh] or "Insulinlispro"[Mesh] or "Insulinaspart"[Mesh] or "Insulin shortactig" [Mesh] or "Insulin isophane"[Mesh] "Insulin detemir" [Mesh] or "Insulin long-acting, human"[Mesh]. Other types of insulin were also included in the search and the complete list of terms was described in the online supplementary material; for all online suppl. material, see www.karger.com/ doi/10.1159/000481355. All potentially eligible studies were considered for review, regardless of the primary outcome, but limited to English, Spanish, French, or Portuguese language. A manual search was also performed in the reference lists of included articles and in recent reviews about the topic.

\section{Study Selection}

All citations retrieved from electronic databases were imported to the EndNote Program. Two reviewers (M.V.V. and L.V.V.) independently analyzed the titles and abstracts of every paper retrieved from the literature search to identify potentially eligible studies. All studies that did not meet the inclusion criteria were excluded. The full text of the remaining papers was obtained for further examination. Disagreements were resolved by a third reviewer (M.J.A.).

The inclusion criteria for the studies were RCTs and cohort studies that evaluated different insulin regimens in patients receiving enteral, parenteral, or both, NS. We excluded studies if patients were on an oral diet without enteral or parenteral support, or were using antihyperglycemic agents but insulin as the only treatment for hyperglycemia, or if they had no documented hyperglycemia.

\section{Data Extraction and Quality Assessment}

The data of included studies were independently extracted by the same 2 reviewers using a standardized data extraction form. Extracted data included the following information: first author's name, year of publication, number of participants, details of the study design (i.e., RCT or observational trial), study duration, and patient characteristics (age, gender, body weight, body mass index, severity of illness score, frequency of patients with diabetes, previous use of insulin or oral antihyperglycemic agents, corticosteroid use, reasons for admission (clinical or surgical), hospitalization setting (ward or ICU), mean baseline blood glucose, and presence of renal replacement therapy. The type of insulin therapy, as well the criteria adopted to initiate insulin therapy, total insulin dose, daily insulin units per body weight, and description of NS were recorded. Total energy, macronutrients, and fiber content were 
also extracted when available. The preestablished evaluated outcomes were glycemic control, hypoglycemia, hospital length of stay, and mortality. Glycemic control targets and hypoglycemia were defined by each study's authors and this information was extracted.

Direct meta-analyses were performed in Stata and indirect meta-analysis was performed in R 3.3.0 (R Foundation for Statistical Computing, Vienna, Austria).

The methodological quality of all included studies was independently assessed (M.V.V. and A.L.J.T.). We used a score based on the Cochrane Collaboration tool to assess risk of bias for RCTs [15] and the Newcastle-Ottawa Scale (NOS) for observational studies [16]. According to the Cochrane Collaboration, biases were classified into 6 domains: selection, performance, detection, attrition, and reporting [17]. The risk of bias for each domain was classified as high, low, or unclear. Regarding the NOS, each study is judged on 8 items and categorized into 3 groups: the selection of the study groups; the comparability of the groups; and the ascertainment of either the exposure or outcome of interest. Up to 9 stars were awarded for each quality item and served as a quick visual assessment [14].

The quality of the body of evidence of each meta-analysis was assessed by the GRADE [18] approach including factors that may decrease (e.g., methodological quality, directness of evidence, heterogeneity, precision of effect estimates, risk of publication bias) or increase (e.g., large magnitude of effect, reduction or spurious effect due to plausible confounding factors, dose-response gradient) the quality of evidence. Each evaluated factor was rated as high, moderate, low, or very low. Using this approach, we considered a serious risk of bias when an individual study had more than 3 unclear or high risk of bias and imprecision was defined as a meta-analysis confidence interval $>0.5$.

\section{Results}

We identified 5,039 studies in our database search (Fig. 1). Of them, 4,885 were excluded based solely on title or abstract, leaving 154 for full text evaluation. From these 154 selected papers, 137 articles were also excluded after full text scrutiny mostly because they used the same regimens of insulin in both arms or due to the absence of a description of NS. Therefore, we included 17 studies (6 RCTs and 11 cohort studies) providing data from 3,260 patients. Clinical trials were heterogeneous in patient populations and there was lack of information on blood glucose variability, duration of blood glucose in target range, caloric and protein intakes, and timing of initiation of nutrition therapy preventing the need for most meta-analysis. Most of the patients $(n=2,657)$ were studied in the ICU. Enteral feeding was the only NS used in 8 studies, parenteral nutrition in 4 , and mixed (enteral plus parenteral) in 5.

The studies included in the current systematic review were classified into 3 groups according to the type of NS

Insulin Regimens to Treat Hyperglycemia

in Hospitalized Patients

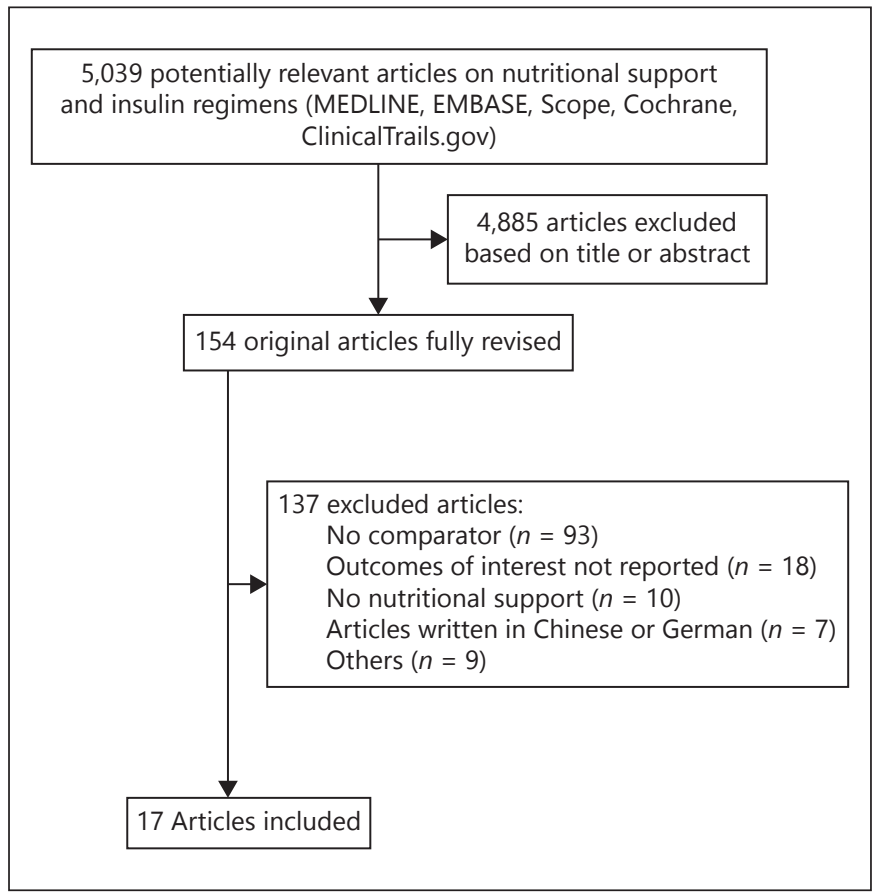

Fig. 1. Flow diagram of literature search to identify studies evaluating different insulin regimens to treat hyperglycemia in hospitalized patients on nutritional support.

received by patients: Enteral NS Group, Parenteral NS Group, and Mixed NS Group.

General features of studies, adopted definitions of hyperglycemia and hypoglycemia, type of insulin regimens, data on mean blood glucose (MBG), and hypoglycemia, besides other studied outcomes for each included study are presented in Table 1 according to the types of NS. The report on outcomes as well as the description or definition of studied variables were not uniform. Mortality was described only in seven studies [19-25] and hospital length of stay in $6[20-22,24,26,27]$. MBG was described in all but one study [28]. The presence of hypoglycemia in the studies was differently reported: number of events, percentage of events, or number of patients with hypoglycemia. Total energy, macronutrients, and fiber content of administered NS were not reported in most of the studies. Details of insulin regimens and available NS information of included studies are presented as online supplementary Table 1.

Enteral NS Group: eight studies [19-21, 28-32] evaluated different insulin regimens for glycemic control in patients receiving enteral support (Table 1a). Two RCTs and 6 cohort studies comprising 1,203 patients were reviewed. Patients' mean age was 64.8 years, $57 \%$ were men, BMI ranged from 28 to $30 \mathrm{~kg} / \mathrm{m}^{2}$, and $64 \%$ had diabetes.

Ann Nutr Metab 2017;71:183-194 185 
Table 1. General characteristics of studies included in the systematic review

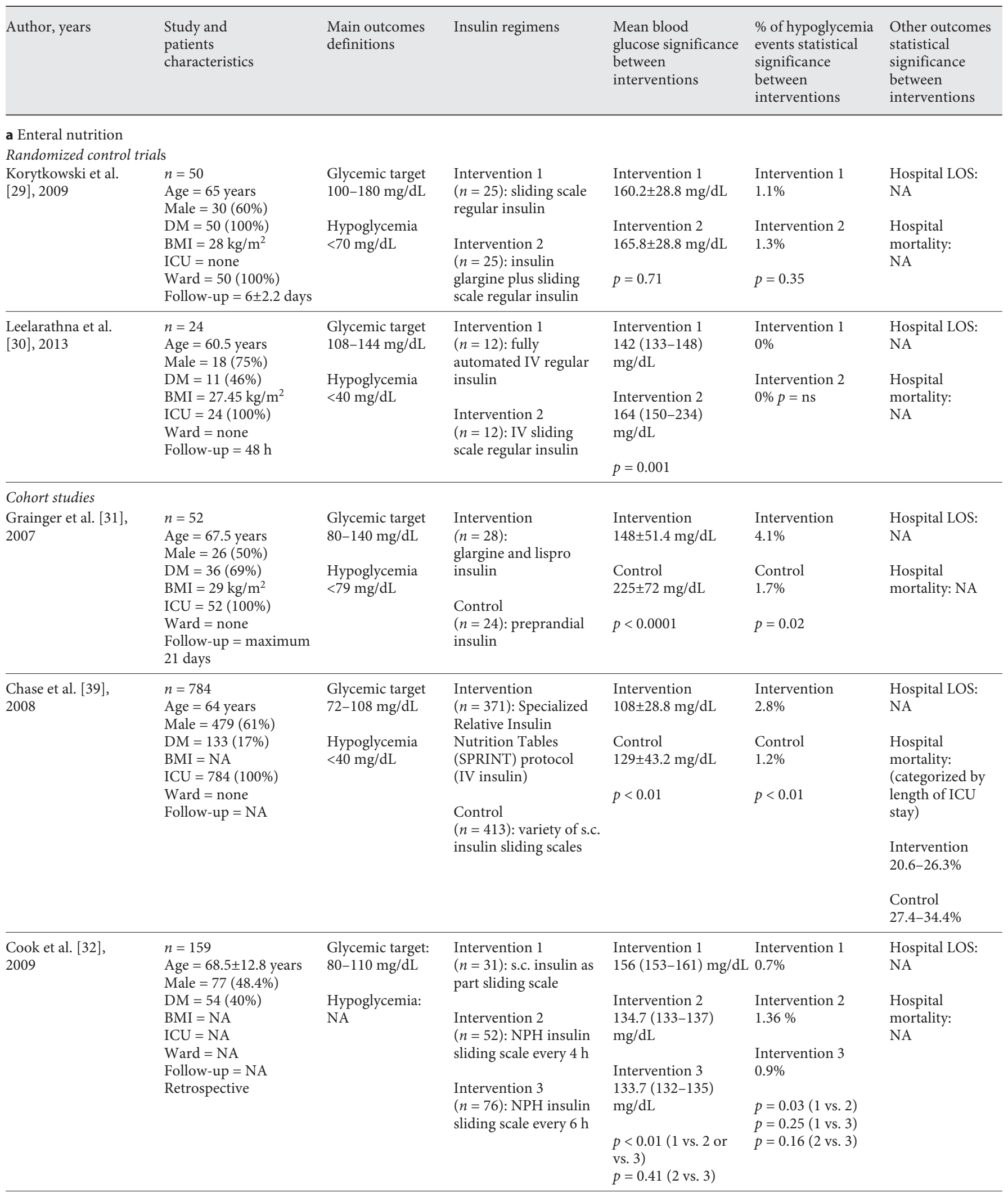


Table 1. (continued)

\begin{tabular}{|c|c|c|c|c|c|c|}
\hline Author, years & $\begin{array}{l}\text { Study and } \\
\text { patients } \\
\text { characteristics }\end{array}$ & $\begin{array}{l}\text { Main outcomes } \\
\text { definitions }\end{array}$ & Insulin regimens & $\begin{array}{l}\text { Mean blood } \\
\text { glucose significance } \\
\text { between } \\
\text { interventions }\end{array}$ & $\begin{array}{l}\% \text { of hypoglycemia } \\
\text { events statistical } \\
\text { significance } \\
\text { between } \\
\text { interventions }\end{array}$ & $\begin{array}{l}\text { Other outcomes } \\
\text { statistical } \\
\text { significance } \\
\text { between } \\
\text { interventions }\end{array}$ \\
\hline Hsia et al. [28], 2011 & $\begin{array}{l}n=22 \\
\text { Age }=23-68 \text { years } \\
\text { Male }=\text { NA } \\
\text { DM }=22(100 \%) \\
\text { BMI }=\text { NA } \\
\text { ICU }=\text { none } \\
\text { Ward = } 100 \% \\
\text { Follow-up }=72 \mathrm{~h} \\
\text { Retrospective }\end{array}$ & $\begin{array}{l}\text { Glycemic target: } \\
\text { 140-180 mg/dL } \\
\text { Hypoglycemia: } \\
<70 \mathrm{mg} / \mathrm{dL}\end{array}$ & $\begin{array}{l}\text { Intervention } 1 \\
(n=8) \text { : basal/bolus } \\
\text { glargine/lispro } \\
\text { Intervention } 2(n=8) \text { : } \\
\text { premixed insulin } \\
70 / 30 \text { twice daily } \\
\text { Intervention } 3 \\
(n=6) \text { : premixed } \\
\text { insulin } 70 / 30 \text { three } \\
\text { times daily }\end{array}$ & $\begin{array}{l}\text { Intervention } 1^{*} \\
24 \% \\
\text { Intervention } 2^{*} \\
22 \% \\
\text { Intervention } 3^{*} \\
69 \% \\
* \% \text { of BG values in } \\
\text { the target range } \\
p<0.01\end{array}$ & $\begin{array}{l}\text { Intervention } 1 \\
5.4 \% \\
\text { Intervention } \\
2.1 \% \\
\text { Intervention } 3 \\
1.4 \% \\
p=0.07 \\
(1 \text { vs. } 2 \text { vs. } 3 \text { ) }\end{array}$ & $\begin{array}{l}\text { Hospital LOS: } \\
\text { NA } \\
\text { Hospital } \\
\text { mortality: } \\
\text { NA }\end{array}$ \\
\hline $\begin{array}{l}\text { Dickerson et al. [20], } \\
2013\end{array}$ & $\begin{array}{l}n=66 \\
\text { Age }=58 \text { years } \\
\text { Male }=17(25 \%) \\
\mathrm{DM}=37(56 \%) \\
\mathrm{BMI}=30 \mathrm{~kg} / \mathrm{m}^{2} \\
\mathrm{ICU}=66(100 \%) \\
\text { Ward = none } \\
\text { Follow-up }=7 \text { days } \\
\text { Retrospective }\end{array}$ & $\begin{array}{l}\text { Glycemic target } \\
70-148 \mathrm{mg} / \mathrm{dL} \\
\text { Hypoglycemia } \\
<40 \mathrm{mg} / \mathrm{dL}\end{array}$ & $\begin{array}{l}\text { Intervention } 1 \\
(n=32) \text { : continuous } \\
\text { IV regular insulin } \\
\text { infusion therapy } \\
\text { Intervention } 2 \\
(n=34) \text { : supplemental } \\
\text { intermittent IV regular } \\
\text { insulin therapy }\end{array}$ & $\begin{array}{l}\text { Intervention } 1 \\
125 \pm 11 \mathrm{mg} / \mathrm{dL} \\
\text { Intervention } 2 \\
133 \pm 14 \mathrm{mg} / \mathrm{dL} \\
p<0.01\end{array}$ & $\begin{array}{l}\text { Intervention } 1^{*} \\
9 \% \\
\text { Intervention } 2^{*} \\
9 \% \\
\text { * Percentage of } \\
\text { patients with any } \\
\text { hypoglycemic } \\
\text { event } \\
\text { p = NA }\end{array}$ & $\begin{array}{l}\text { Hospital LOS: } \\
\text { Intervention } 1 \\
41 \pm 27 \text { days } \\
\text { Intervention } 2 \\
39 \pm 28 \text { days } \\
p=\mathrm{ns} \\
\text { Hospital } \\
\text { mortality: } \\
\text { Intervention } 1 \\
3.2 \% \\
\text { Intervention } 2 \\
13.3 \% \\
p=\mathrm{ns}\end{array}$ \\
\hline $\begin{array}{l}\text { Murphy et al. [21], } \\
2014\end{array}$ & $\begin{array}{l}n=46 \\
\text { Age }=74.8 \text { years } \\
\text { Male }=27(58.6 \%) \\
\text { DM }=46(100 \%) \\
\text { BMI = NA } \\
\text { ICU = NA } \\
\text { Ward = NA } \\
\text { Follow-up = over } \\
40 \text { days } \\
\text { Retrospective }\end{array}$ & $\begin{array}{l}\text { Glycemic target } \\
\text { NA } \\
\text { Hypoglycemia } \\
<72 \mathrm{mg} / \mathrm{dL}\end{array}$ & $\begin{array}{l}\text { Intervention } 1 \\
(n=18) \text { : premixed } \\
\text { insulin } 70 / 30 \\
\text { Intervention } 2 \\
(n=13) \text { : short acting } \\
\text { s.c. insulin before each } \\
\text { feed plus Glargine } \\
\text { insulin at night } \\
\text { Intervention } 3 \\
\text { ( } n=15) \text { : long acting } \\
\text { analogue insulin }\end{array}$ & $\begin{array}{l}\text { Intervention } 1 \\
227 \pm 97.3 \mathrm{mg} / \mathrm{dL} \\
\text { Intervention } 2 \\
225 \pm 76 \mathrm{mg} / \mathrm{dL} \\
\text { Intervention } 3 \\
218 \pm 90 \mathrm{mg} / \mathrm{dL} \\
p=0.46\end{array}$ & $\begin{array}{l}\text { Intervention } 1^{*} \\
4 \% \\
\text { Intervention } 2^{*} \\
0 \% \\
\text { Intervention } 3^{*} \\
7 \% \\
* \% \text { hypoglycemia } \\
\text { during feeding } \\
p=0.004\end{array}$ & $\begin{array}{l}\text { Hospital LOS: } \\
\text { Over } 40 \text { days for } \\
\text { the } 3 \text { groups } \\
\text { Hospital } \\
\text { Mortality: } \\
\text { Intervention } 1 \\
3 \text { (16.7\%) } \\
\text { Intervention } 2 \\
4 \text { (30.8\%) } \\
\text { Intervention } 3 \\
5 \text { (33.8\%) } \\
p=\text { NA }\end{array}$ \\
\hline $\begin{array}{l}\text { b Parenteral nutrition } \\
\text { Randomized trials } \\
\text { Hakeam et al. [33], } \\
2016\end{array}$ & $\begin{array}{l}n=67 \\
\text { Age }=58 \text { years } \\
\text { Male }=28(42 \%) \\
\text { DM }=55(82 \%) \\
\text { BMI }=27.7 \mathrm{~kg} / \mathrm{m}^{2} \\
\text { ICU }=\text { none } \\
\text { Ward }=67(100 \%) \\
\text { Follow-up }=\text { NA }\end{array}$ & $\begin{array}{l}\text { Glycemic target } \\
\text { 140-180 mg/dL } \\
\text { Hypoglycemia } \\
<70 \mathrm{mg} / \mathrm{dL}\end{array}$ & $\begin{array}{l}\text { Intervention } \\
(n=35) \text { : Glargine } \\
\text { insulin at night } \\
\text { Control } \\
(n=32) \text { : regular } \\
\text { insulin added to } \\
\text { parenteral nutrition }\end{array}$ & $\begin{array}{l}\text { Intervention } \\
136.8 \pm 39.6 \mathrm{mg} / \mathrm{dl} \\
\text { Control } \\
148 \pm 37.8 \mathrm{mg} / \mathrm{dL} \\
p=0.39\end{array}$ & $\begin{array}{l}\text { Intervention* } \\
5.7 \% \\
\text { Control* } \\
6.25 \% \\
* \% \text { of patients with } \\
\text { hypoglycemia } \\
p>0.1\end{array}$ & $\begin{array}{l}\text { Hospital LOS: } \\
\text { NA } \\
\text { Hospital } \\
\text { Mortality: } \\
\text { NA }\end{array}$ \\
\hline
\end{tabular}


Table 1. (continued)

\begin{tabular}{|c|c|c|c|c|c|c|}
\hline Author, years & $\begin{array}{l}\text { Study and } \\
\text { patients } \\
\text { characteristics }\end{array}$ & $\begin{array}{l}\text { Main outcomes } \\
\text { definitions }\end{array}$ & Insulin regimens & $\begin{array}{l}\text { Mean blood } \\
\text { glucose significance } \\
\text { between } \\
\text { interventions }\end{array}$ & $\begin{array}{l}\% \text { of hypoglycemia } \\
\text { events statistical } \\
\text { significance } \\
\text { between } \\
\text { interventions }\end{array}$ & $\begin{array}{l}\text { Other outcomes } \\
\text { statistical } \\
\text { significance } \\
\text { between } \\
\text { interventions }\end{array}$ \\
\hline $\begin{array}{l}\text { Oghazian et al. [26], } \\
2015\end{array}$ & $\begin{array}{l}n=42 \\
\text { Age }=56.1 \text { years } \\
\text { Male }=26(43 \%) \\
\text { DM }=0 \\
\text { BMI }=23.5 \mathrm{~kg} / \mathrm{m}^{2} \\
\text { ICU }=100 \% \\
\text { Ward = none } \\
\text { Follow-up = NA }\end{array}$ & $\begin{array}{l}\text { Glycemic target } \\
110-180 \mathrm{mg} / \mathrm{dL} \\
\text { Hypoglycemia } \\
<700 \mathrm{mg} / \mathrm{dL}\end{array}$ & $\begin{array}{l}\text { Intervention } \\
(n=21) \text { : Glargine } \\
\text { insulin } \\
\text { Control } \\
(n=21) \text { : regular insulin } \\
\text { added to parenteral } \\
\text { nutrition bag }\end{array}$ & $\begin{array}{l}\text { Intervention } \\
140 \pm 19 \mathrm{mg} / \mathrm{dl} \\
\text { Control } \\
142 \pm 15 \mathrm{mg} / \mathrm{dL} \\
p=0.741\end{array}$ & $\begin{array}{l}\text { Intervention* } \\
19 \% \\
\text { Control* } \\
0 \% \\
\text { *\% of patients with } \\
\text { hypoglycemia } \\
p=0.107\end{array}$ & $\begin{array}{l}\text { Hospital LOS: } \\
\text { Intervention } \\
37 \text { days } \\
\text { Control } \\
37 \text { days } \\
\text { Survival to } \\
\text { hospital } \\
\text { discharge: NA }\end{array}$ \\
\hline $\begin{array}{l}\text { Cohort studies } \\
\text { Jakoby et al. [34], } 2012\end{array}$ & $\begin{array}{l}n=48 \\
\text { Age }=58.5 \text { years } \\
\text { Male }=21(43.8 \%) \\
\text { DM }=16(33.3 \%) \\
\text { BMI = NA } \\
\text { ICU = } 10(20.8 \%) \\
\text { Ward = } 38(79.1 \%) \\
\text { Follow-up = NA } \\
\text { Prospective }\end{array}$ & $\begin{array}{l}\text { Glycemic target } \\
140 \mathrm{mg} / \mathrm{dL} \\
\text { Hypoglycemia: } \\
<80 \mathrm{mg} / \mathrm{dL}\end{array}$ & $\begin{array}{l}\text { Intervention } \\
(n=22) \text { : insulin doses } \\
\text { based on carbohydrate } \\
\text { delivery (regular insulin } \\
\text { in parenteral nutrition } \\
\text { bag and NPH) } \\
\text { Control } \\
\text { ( } n=26) \text { : ad hoc insulin } \\
\text { management (NPH, } \\
\text { glargine, etc.) }\end{array}$ & $\begin{array}{l}\text { Intervention } \\
138 \pm 37 \mathrm{mg} / \mathrm{dL} \\
\text { Control } 159 \pm 46 \\
\mathrm{mg} / \mathrm{dL} \\
p<0.0001\end{array}$ & $\begin{array}{l}\text { Intervention* } \\
3 \% \\
\text { Control* }^{*} \\
1 \% \\
\text { * Number of } \\
\text { glycemic events } \\
<40 \mathrm{mg} / \mathrm{dL} \\
p=0.12\end{array}$ & $\begin{array}{l}\text { Hospital LOS: } \\
\text { NA } \\
\text { Hospital } \\
\text { mortality: } \\
\text { NA }\end{array}$ \\
\hline Neff et al. [22], 2014 & $\begin{array}{l}n=53 \\
\text { Age }=68 \text { years } \\
\text { Male }=30(56.6 \%) \\
\mathrm{DM}=18(33.9 \%) \\
\mathrm{BMI}=27 \mathrm{~kg} / \mathrm{m}^{2} \\
\mathrm{ICU}=\text { none } \\
\text { Ward = 53 }(100 \%) \\
\text { Follow-up = NA } \\
\text { Retrospective }\end{array}$ & $\begin{array}{l}\text { Glycemic target } \\
72-180 \mathrm{mg} / \mathrm{dL} \\
\text { Hypoglycemia } \\
<72 \mathrm{mg} / \mathrm{dL}\end{array}$ & $\begin{array}{l}\text { Intervention } \\
(n=32): \text { IV insulin } \\
\text { protocol } \\
\text { Control } \\
(n=21) \text { : individually } \\
\text { prescribed s.c. insulin } \\
\text { supplemental scales } \\
\text { (basal + rapid acting } \\
\text { analogues) }\end{array}$ & $\begin{array}{l}\text { Intervention } \\
173 \pm 37 \mathrm{mg} / \mathrm{dL} \\
\text { Control } \\
202 \pm 48 \mathrm{mg} / \mathrm{dL} \\
p=0.009\end{array}$ & $\begin{array}{l}\text { Intervention* } \\
40 \% \\
\text { Control* }^{*} \\
29 \% \\
\text { * Percentage of } \\
\text { patients with any } \\
\text { hypoglycemic } \\
\text { event } \\
p=0.19\end{array}$ & $\begin{array}{l}\text { Hospital LOS: } \\
\text { Intervention } \\
61 \pm 49 \text { days } \\
\text { Control } \\
43 \pm 35 \text { days } \\
p=0.08 \\
\text { Survival to } \\
\text { hospital } \\
\text { discharge: } \\
\text { Intervention } \\
77 \% \\
\text { Control } \\
67 \% \\
p=0.2\end{array}$ \\
\hline $\begin{array}{l}\text { c Mixed nutritional sup } \\
\text { Randomized trials } \\
\text { Holzinger et al. [23], } \\
2004\end{array}$ & $\begin{array}{l}n=40 \\
\text { Age }=54.5 \text { years } \\
\text { Male }=27(67.5 \%) \\
\mathrm{DM}=\text { none } \\
\mathrm{BMI}=26.2 \mathrm{~kg} / \mathrm{m}^{2} \\
\mathrm{ICU}=40(100 \%) \\
\text { Ward }=\text { none } \\
\text { Follow-up }=9 \pm 4.5 \\
\text { days }\end{array}$ & $\begin{array}{l}\text { Glycemic target: } \\
\text { NA } \\
\text { Hypoglycemia: } \\
\text { NA }\end{array}$ & $\begin{array}{l}\text { Intervention } \\
(n=20): \text { low dose } \\
\text { continuous IV insulin } \\
(1 \mathrm{IU} / \mathrm{h}) \\
\text { Control } \\
(n=20) \text { : placebo }\end{array}$ & $\begin{array}{l}\text { Intervention } \\
131 \pm 39 \mathrm{mg} / \mathrm{dL} \\
\text { Control } \\
128 \pm 30 \mathrm{mg} / \mathrm{dL} \\
p=\mathrm{ns}\end{array}$ & $\begin{array}{l}\text { Intervention } \\
0 \% \\
\text { Control } \\
0 \% \\
p=\mathrm{ns}\end{array}$ & $\begin{array}{l}\text { Hospital } \\
\text { LOS: } \\
\text { NA } \\
\text { Hospital } \\
\text { mortality: } \\
\text { Intervention } \\
5(25 \%) \\
\text { Control } \\
6(30 \%) \\
p=\mathrm{ns}\end{array}$ \\
\hline
\end{tabular}


Table 1. (continued)

\begin{tabular}{|c|c|c|c|c|c|c|}
\hline Author, years & $\begin{array}{l}\text { Study and } \\
\text { patients } \\
\text { characteristics }\end{array}$ & $\begin{array}{l}\text { Main outcomes } \\
\text { definitions }\end{array}$ & Insulin regimens & $\begin{array}{l}\text { Mean blood } \\
\text { glucose significance } \\
\text { between } \\
\text { interventions }\end{array}$ & $\begin{array}{l}\% \text { of hypoglycemia } \\
\text { events statistical } \\
\text { significance } \\
\text { between } \\
\text { interventions }\end{array}$ & $\begin{array}{l}\text { Other outcomes } \\
\text { statistical } \\
\text { significance } \\
\text { between } \\
\text { interventions }\end{array}$ \\
\hline $\begin{array}{l}\text { Van Herpe et al. [24], } \\
2013\end{array}$ & $\begin{array}{l}n=300 \\
\text { Age }=63.5 \pm 14.5 \text { years } \\
\text { Male: } 181(60 \%) \\
\mathrm{DM}=64(21.3 \%) \\
\mathrm{BMI}=27.7 \pm 5.15 \\
\mathrm{~kg} / \mathrm{m}^{2} \\
\mathrm{ICU}=300(100 \%) \\
\text { Ward = none } \\
\text { Follow-up }=14 \\
\text { days }\end{array}$ & $\begin{array}{l}\text { Glycemic target } \\
80-110 \mathrm{mg} / \mathrm{dL} \\
\text { Hypoglycemia } \\
<40 \mathrm{mg} / \mathrm{dL}\end{array}$ & $\begin{array}{l}\text { Intervention } 1 \\
(n=149) \text { : algorithm } \\
\text { guided IV insulin } \\
\text { Intervention } 2 \\
(n=151) \text { : } \\
\text { nurse-directed IV } \\
\text { insulin }\end{array}$ & $\begin{array}{l}\text { Intervention } 1 \\
106 \pm 9 \mathrm{mg} / \mathrm{dL} \\
\text { Intervention } 2 \\
107 \pm 11 \mathrm{mg} / \mathrm{dL} \\
p=0.36\end{array}$ & $\begin{array}{l}\text { Intervention } 1 \\
0 \% \\
\text { Intervention } 2 \\
6(0.1 \%) \\
p=0.015\end{array}$ & $\begin{array}{l}\text { Hospital LOS: } \\
\text { Intervention } 1 \\
16 \text { (10-33) days } \\
\text { Intervention } 2 \\
14(9-27) \text { days } \\
p=0.24 \\
\text { Hospital } \\
\text { mortality: } \\
\text { Intervention } 1 \\
19 \text { (12.8\%) } \\
\text { Intervention } 2 \\
10 \text { ( } 6.6 \%) \\
p=\text { ns }\end{array}$ \\
\hline $\begin{array}{l}\text { Cohort studies } \\
\text { Thomas et al. [35], } \\
2005\end{array}$ & $\begin{array}{l}n=891 \\
\text { Age }=51.4 \text { years } \\
\text { Male = } 458(56 \%) \\
\text { DM = NA } \\
\text { BMI = NA } \\
\text { ICU = } 891(100 \%) \\
\text { Ward = none } \\
\text { Follow-up = NA } \\
\text { Retrospective }\end{array}$ & $\begin{array}{l}\text { Glycemic target: } \\
\text { maximum } 128 \\
\text { mg/dL } \\
\text { Hypoglycemia: } \\
<50 \mathrm{mg} / \mathrm{dL}\end{array}$ & $\begin{array}{l}\text { Control } \\
(n=288) \text { : no protocol } \\
\text { Intervention } 1 \\
(n=502) \text { : protocol-IV } \\
\text { insulin } \\
\text { Intervention } 2 \\
(n=101) \text { : modified } \\
\text { protocol of IV insulin }\end{array}$ & $\begin{array}{l}\text { Control: } 131 \pm 32.4 \\
\mathrm{mg} / \mathrm{dL} \\
\text { Intervention } 1 \text { : } \\
118 \pm 28.8 \\
\text { Intervention } 2 \text { : } \\
111.6 \pm 23.4 \\
p=\mathrm{NA}\end{array}$ & $\begin{array}{l}\text { Control* } \\
1 \\
\text { Intervention } 1^{*} \\
13 \\
\text { Intervention } 2^{*} \\
6 \\
* \text { Number of } \\
\text { hypoglycemic } \\
\text { events } \\
p=\mathrm{NA}\end{array}$ & $\begin{array}{l}\text { Hospital LOS: } \\
\text { NA } \\
\text { ICU mortality: } \\
\text { Control: } 76(26 \%) \\
\text { Intervention 1: } \\
125 \text { (25\%) } \\
\text { Intervention 2: } 27 \\
(27 \%) \\
p=\text { NA }\end{array}$ \\
\hline $\begin{array}{l}\text { Dortch et al. [25], } \\
2008\end{array}$ & $\begin{array}{l}n=552 \\
\text { Age }=41 \text { years } \\
\text { Male }=391(71 \%) \\
\mathrm{DM}=40(7.2 \%) \\
\mathrm{BMI}=\mathrm{NA} \\
\mathrm{ICU}=552(100 \%) \\
\text { Ward = none } \\
\text { Follow-up = NA }\end{array}$ & $\begin{array}{l}\text { Glycemic target: } \\
80-110 \mathrm{mg} / \mathrm{dL} \\
\text { Hypoglycemia: } \\
<40 \mathrm{mg} / \mathrm{dL}\end{array}$ & $\begin{array}{l}\text { Intervention } \\
(n=243) \text { : IV insulin } \\
\text { computerized protocol } \\
\text { Control }(n=309) \text { : } \\
\text { paper-based IV insulin } \\
\text { protocol }\end{array}$ & $\begin{array}{l}\text { Intervention } \\
116 \pm 37 \mathrm{mg} / \mathrm{dL} \\
\text { Control } \\
120 \pm 37 \mathrm{mg} / \mathrm{dL} \\
p<0.001\end{array}$ & $\begin{array}{l}\text { Intervention } \\
0.54 \% \\
\text { Control } \\
0.23 \% \\
p<0.001\end{array}$ & $\begin{array}{l}\text { Hospital LOS: } \\
\text { NA } \\
\text { Hospital } \\
\text { mortality: } \\
\text { Intervention } \\
33(13.6 \%) \\
\text { Control } \\
51(16.5 \%) \\
p=0.4\end{array}$ \\
\hline $\begin{array}{l}\text { Dickerson et al. [27], } \\
2014\end{array}$ & $\begin{array}{l}n=46 \\
\text { Age }=68 \text { years } \\
\text { Male }=38(82.6 \%) \\
\mathrm{DM}=20(43.5 \%) \\
\mathrm{BMI}=33 \pm 8.5 \mathrm{~kg} / \mathrm{m}^{2} \\
\mathrm{ICU}=46(100 \%) \\
\text { Ward = none } \\
\text { Follow-up }=7 \text { days }\end{array}$ & $\begin{array}{l}\text { Glycemic target } \\
150-180 \mathrm{mg} / \mathrm{dL} \\
\text { Hypoglycemia } \\
<40 \mathrm{mg} / \mathrm{dL}\end{array}$ & $\begin{array}{l}\text { Intervention } 1 \\
(n=21) \text { : former } \\
\text { protocol } \\
\text { Intervention } 2 \\
(n=25) \text { : new protocol }\end{array}$ & $\begin{array}{l}\text { Intervention } 1 \\
145 \pm 10 \mathrm{mg} / \mathrm{dL} \\
\text { Intervention } 2 \\
133 \pm 14 \mathrm{mg} / \mathrm{dL} \\
p<0.001\end{array}$ & $\begin{array}{l}\text { Intervention } 1 \\
0.35 \% \\
\text { Intervention } 2 \\
0 \% \\
p=0.005\end{array}$ & $\begin{array}{l}\text { Hospital LOS: } \\
\text { Intervention } 1 \\
38 \pm 26 \text { days } \\
\text { Intervention } 2 \\
44 \pm 35 \text { days } \\
p=\text { ns } \\
\text { Hospital } \\
\text { mortality: NA }\end{array}$ \\
\hline
\end{tabular}

BMI, body mass index; DM, diabetes mellitus; ICU, intensive care unit; IV, intravenous; NA, not available; LOS, length of stay; ns, not significant; s.c. = subcutaneous. Data are shown as mean $\pm \mathrm{SD}$; median (interquartile range). 


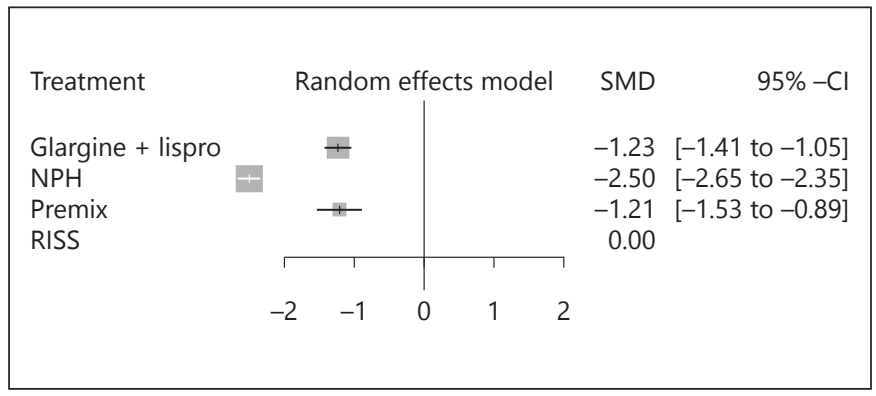

Fig. 2. Forest plot (indirect meta-analysis, random-effect model) of rapid insulin sliding scale (RISS), NPH, Premix, Glargine + Lispro insulin regimen effects on mean blood glucose $(\mathrm{mg} / \mathrm{dL})$ in hospitalized patients on enteral support.

Half of the studies were conducted in ICU settings and 3 studies did not inform the patients' setting. Glycemic targets and hypoglycemia definitions were heterogeneous: the glycemic target ranged from 70 to $180 \mathrm{mg} / \mathrm{dL}$ and hypoglycemia was defined as capillary glucose less than 40 $\mathrm{mg} / \mathrm{dL}$ in 3 studies $[19,20,30]$, less than $70 \mathrm{mg} / \mathrm{dL}$ in 3 $[21,28,29]$ and less than $79 \mathrm{mg} / \mathrm{dL}$ in one [31]. Only one study had no definition for hypoglycemia [32]. Only one study in this category presented data about hospital length of stay [20] and 3 described hospital mortality [19-21]. MBG control ranged from 108 to $225 \mathrm{mg} / \mathrm{dL}$ and was $<180 \mathrm{mg} / \mathrm{dL}$ in $62.5 \%$ of the studies (5 out of 8 ) in both control and intervention groups. The percentage of hypoglycemia varied from 0 to $9 \%$. The most used insulin regimen to control glucose was sliding scale insulin $[19,29$, $31,32]$. Regarding enteral support, 4 studies [19, 20, 31, 32] used special formulas (online suppl. Table 1a).

Figure 2 summarizes the results of the indirect metaanalysis $[21,31,32]$ comparing all evaluated insulin regimens. NPH insulin ranked as the best regimen to reduce MBG level (SMD 95\% CI): NPH, $-2.50 \mathrm{mg} / \mathrm{dL}$ ( -2.65 to $-2.35)$; Premix, -1.21 (-1.53 to -0.89$)$, and Glargine + Lispro, -1.23 (-1.41 to -1.05$) \mathrm{mg} / \mathrm{dL}$ as compared to sliding scale rapid insulin regimen. There was no difference between Premix and Glargine + Lispro insulin regimens.

Parenteral NS Group: four studies [22, 26, 33, 34] evaluated different insulin regimens for glycemic control in parenteral support (Table 1b). Two RCTs and 2 cohort studies comprising 228 patients were reviewed. Mean age was 60.7 years, BMI $23.5-27.7 \mathrm{~kg} / \mathrm{m}^{2}$, and $40 \%$ of the patients had diabetes. One study was conducted exclusively in an ICU setting [26], 2 studies included exclusively ward patients [22,33], and one study included patients from ICU and ward settings [34]. Glycemic targets and hypoglycemia definitions were heterogeneous in these studies: the maximum glycemic target ranged from 140 to 180 $\mathrm{mg} / \mathrm{dL}$ and hypoglycemia was defined as glucose less than $80 \mathrm{mg} / \mathrm{dL}$ in 2 studies and around $70 \mathrm{mg} / \mathrm{dL}$ in 2 others. MBG ranged from 137 to $173 \mathrm{mg} / \mathrm{dL}$ in the intervention group and from 142 to $202 \mathrm{mg} / \mathrm{dL}$ in the control group. The percentage of hypoglycemia varied from 3 to $40 \%$ in the intervention group and from 0 to $29 \%$ in the control group. MBG was $<180 \mathrm{mg} / \mathrm{dL}$ both in control and intervention groups, except in one study [22].

RCTs compared subcutaneous insulin glargine with regular insulin added to the parenteral nutrition bag $[26,33]$. Meta-analyses did not demonstrate differences in MBG (SMD 95\% CI $-3.78 \mathrm{mg} / \mathrm{dL}[-11.93$ to -4.37$\left.] ; I^{2}=0 \%\right]$ or frequency of hypoglycemia (RR 95\% CI 2.48 [0.61-10.10]; $I^{2}=45.6 \%$ ) between the 2 insulin regimens (Fig. 3a, b).

Mixed NS Group (enteral plus parenteral): five studies $[23-25,27,35]$ evaluated different insulin regimens for glycemic control in mixed (parenteral plus enteral) NS (Table 1c). Two RCTs and 3 cohort studies comprising 1,829 patients were reviewed. All studies were conducted in the ICU. One study was conducted exclusively with patients with acute kidney failure [27] and one study excluded patients with diabetes [23]. The mean age was 50.5 years, BMI $25.7-27 \mathrm{~kg} / \mathrm{m}^{2}$, and $18 \%$ of patients had diabetes. Glycemic targets and hypoglycemia definitions were diverse: the maximum glycemic target ranged from 80 to $180 \mathrm{mg} / \mathrm{dL}$ and hypoglycemia was defined as glucose less than $40 \mathrm{mg} / \mathrm{dL}$ in 3 studies [24, 25, 27]. MBG ranged from 106 to $145 \mathrm{mg} / \mathrm{dL}$ in the intervention group and from 107 to $133 \mathrm{mg} / \mathrm{dL}$ in the control group. Hypoglycemic events varied from zero to less than $1 \%$ in the included studies. In all studies, MBG was lower than $180 \mathrm{mg} / \mathrm{dL}$.

Three studies compared computer-based protocols with standard protocols for hyperglycemia control (one RCT and 2 cohort studies) [24, 25, 35]. The RCT [24] protocol took into account several variables (patient profile at admission, blood glucose, insulin dose, nutrition, and steroid medication) and was not comparable to cohort study protocols. A meta-analysis of cohort studies $[25,35]$ demonstrated that the MBG value was higher in paper-based than computer-based protocols (MD 95\% CI $\left.5.41[1.40-9.40] ; I^{2}=0 \%\right)$. Descriptions of hypoglycemic events were not comparable in studies.

\section{Quality Evaluation}

Individual quality of RCTs revealed a low risk of bias for most evaluated domains, except performance (bias for most of studies; online suppl. Table 2). For observational studies, NOS scored moderate (4-7 stars out of 9; online suppl. Table 3).
Verçoza Viana/Verçoza Viana/Tavares/ de Azevedo 


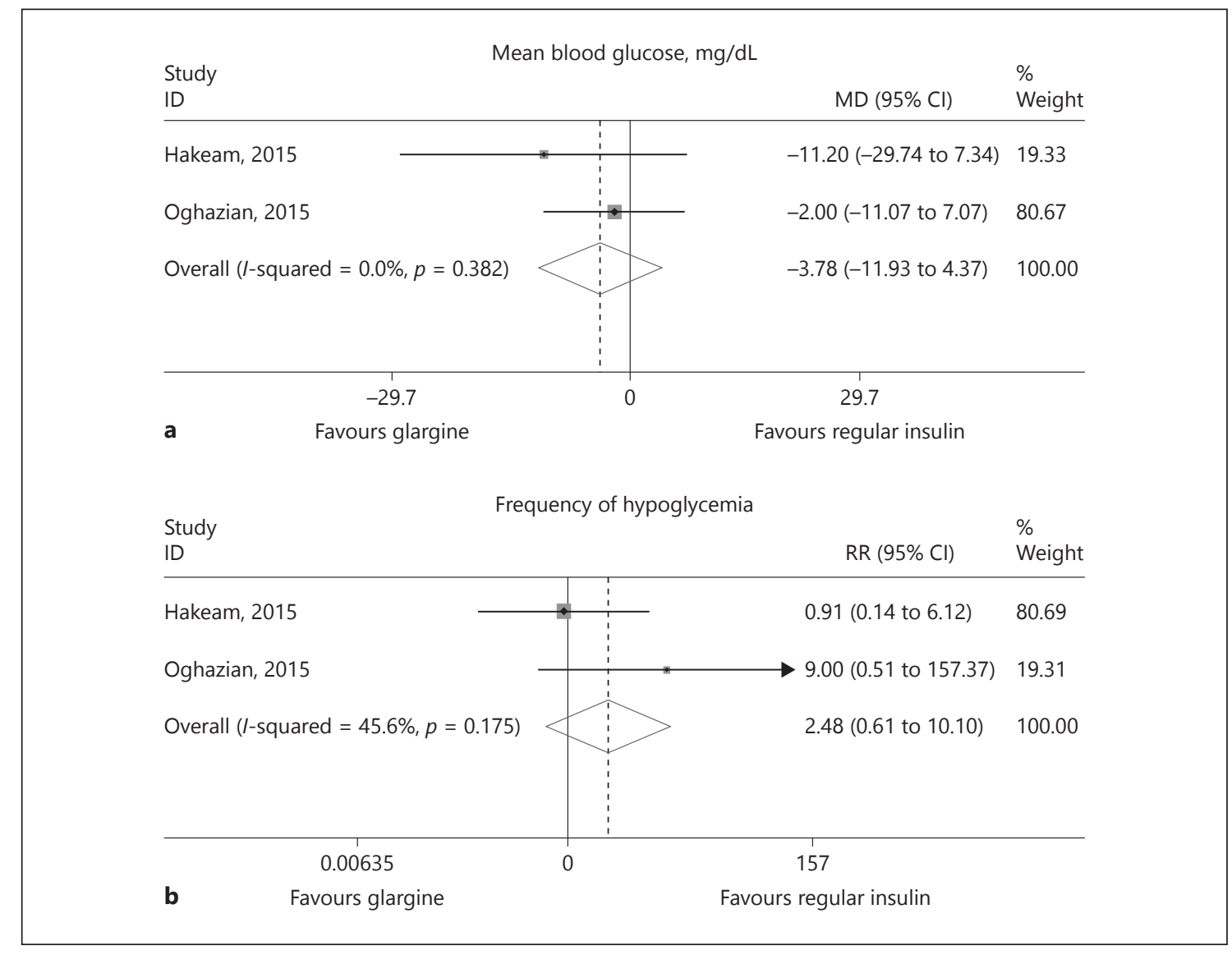

Fig. 3. Forest plots (meta-analyses, random-effects models) of the effect of different insulin regimens on mean blood glucose (a) and on the frequency of hypoglycemia in parenteral nutrition support (b).

The GRADE quality of evidence for systematic review was evaluated (online suppl. Table 4). In the indirect meta-analysis of the insulin regimens used to treat hyperglycemia in patients on enteral support, the GRADE score evidence was considered very low due to the indirect nature of the comparison and small number of studies. The quality of direct meta-analyses of insulin regimens on parenteral nutrition support was moderate both for MBG and frequency of hypoglycemia events. The quality of evidence was also low for meta-analysis of MBG of insulinbased protocol regimens in mixed NS.

\section{Discussion}

This systematic review included 17 studies, 6 RCTs, and 11 cohort studies, designed to evaluate diverse insulin regimens used to treat hyperglycemia and comprised 3,260 hospitalized patients receiving enteral, parenteral, or mixed NS. MBG ranged from 106 to $225 \mathrm{mg} / \mathrm{dL}$ and frequency of hypoglycemia from 0 to $40 \%$. Taking into account the type of administered NS, we performed some direct or indirect meta-analyses. These comparisons allowed us to demonstrate that in patients on enteral support, the MBG was lower with NPH insulin than with sliding scale insulin, Premix, or Glargine + Lispro regimens (indirect meta-analysis). On the other hand, for parenteral nutrition support, the reduction patterns of hyperglycemia did not differ when insulin was added to the parenteral nutrition bag or with subcutaneous insulin glargine use. Lastly, for hyperglycemic patients on mixed NS insulin regimens using computer-based protocols seemed to promote more MBG reduction than paper protocols. Hypoglycemia could be compared only in patients on parenteral nutrition in whom hyperglycemia was treated using regular insulin added to the parenteral nutrition bag or with subcutaneous insulin glargine and no difference was observed between these insulin regimens. 
However, regardless of the insulin regimen used, there was only a small, not clinically significant, reduction in MBG.

Hyperglycemia in critically ill patients may be an adaptive response to spare glucose to brain, erythrocytes, and injured tissues, although the benefits of this response may be outweighed by detrimental effects over the long term [36]. Hence, there is still some controversy about the degree of glycemic control in hospitalized patients, especially in ICU settings $[6,7]$. Most critical care and endocrinology societies nowadays endorse a random glucose value below $180 \mathrm{mg} / \mathrm{dL}[4,30]$ as the target for hospitalized patients. In our systematic review, there was a great variability in glycemic target even within the nutritional categories; however, in most studies (76\%), MBG values were in agreement with current recommendations. This MBG goal was reached irrespective of the insulin regimen used to treat hyperglycemia. Only in 4 studies, 3 conducted in ICU settings, the target of strict glucose control was less than $110 \mathrm{mg} / \mathrm{dL}[19,24,25,32]$, but MBG reached this goal only in half of them $[19,24]$. Actually the differences between MBG reductions of insulin interventions were lower than $6 \mathrm{mg} / \mathrm{dL}$. This small difference in MBG would require a very large study to show a clinical benefit. Therefore, it should be emphasized that although our results on glucose control were statistically significant, there is no clinical relevance.

A post hoc analysis of the NICE-SUGAR trial revealed that hypoglycemia was strongly associated with mortality [37] and guidelines recommend that insulin infusion programs have to be coordinated with NS intervention to minimize the risk of both hyperglycemia and hypoglycemia [11]. In this sense, severe hypoglycemia, defined as blood glucose lower than $40 \mathrm{mg} / \mathrm{dL}$ [38], is the most feared adverse effect related to insulin treatment. Therefore, the choice of insulin regimen should also be based on the absence of harmful effects. From the total of 17 included studies, only $6[20,24,25,27,30,39]$ reported data on severe hypoglycemia and its frequency varied from 0 to $2.8 \%$. Moreover, in the current systematic review, the definition of hypoglycemia was heterogeneous and unclear in most studies. We could compare only the frequency of hypoglycemia in 2 studies $[26,33]$ conducted in patients on parenteral nutrition and there was no difference in hypoglycemic events.

Our systematic review had some limitations even though we have performed a comprehensive search and detailed data extraction strictly following all current recommended guidelines $[14,15,40]$. It was not possible to compare most of the studies due to their methodological differences, unclear or different outcomes definitions, or even missing data. The quality of included studies was moderate and most of them were observational. Very few studies evaluated mortality and hospital length of stay and, therefore, these outcomes could not be compared. Heterogeneity of insulin regimens did not allow us to perform direct comparisons for other predetermined outcomes. The differences among the individual studies are likely to be important. The type of patients (medical, surgical) and the place where the studies occurred (ICU or ward) differed significantly and this might influence the optimal glycemic target. Lack of clinical outcomes could have been attributed to heterogeneity in patient population between studies, differing populations whereby benefit from level of glycemic control may be different, blood variability, hours/day in target blood glucose range, caloric and protein intakes, and timing of initiation of nutrition therapy. Also, samples with diverse characteristics, such as patients with or without diabetes, were studied together. The possible influence of this heterogeneity on our results is unknown, since sensitivity analyses were not allowed due to the characteristics of these studies. Lastly, NS was heterogeneous even within the same category and poorly described and this might be a potential confounder factor.

According to our systematic review, studies comparing different insulin regimens to treat hyperglycemia in hospitalized patients requiring NS are heterogeneous. Our best results on reduction of MBG obtained by insulin regimens using NPH in enteral nutrition or rapid insulin computer-based protocols in mixed NS were not clinically relevant. Additional RCTs, with focus on hard outcomes and severe hypoglycemia, beyond hyperglycemia per se, are needed. Possibly, the best choice might be the less expensive insulin regimen with less associated hypoglycemia events. However, until now it has not been feasible to establish the best insulin regimen to treat hyperglycemia in hospitalized patients on NS.

\section{Acknowledgments}

We acknowledge the support received from Fundação de Amparo a Pesquisa do Rio Grande do Sul. This manuscript is dedicated to the memory of our dear friend, colleague, and co-author Mirela Jobim de Azevedo, MD, PhD who tragically passed away in May 2017.

\section{Disclosure Statement}

The authors declare no conflicts of interest.
192

Ann Nutr Metab 2017;71:183-194 DOI: $10.1159 / 000481355$
Verçoza Viana/Verçoza Viana/Tavares/ de Azevedo 


\section{Author Contribution}

Study concept and design: M.V.V., L.V.V., and M.J.A. Literature search and data extraction: M.V.V., L.V.V., and A.L.T. Assessment of methodological quality: M.V.V. and A.L.T. Statistical analysis: L.V.V. and M.V.V. Manuscript drafting: all authors. Critical revision of the manuscript for important intellectual content: all authors.

\section{References}

1 Farrokhi F, Smiley D, Umpierrez GE: Glycemic control in non-diabetic critically ill patients. Best Pract Res Clin Endocrinol Metab 2011;25:813-824.

2 Viana MV, Moraes RB, Fabbrin AR, Santos MF, Gerchman F: [Assessment and treatment of hyperglycemia in critically ill patients]. Rev Bras Ter Intensiva 2014;26:71-76.

3 Souba WW: Nutritional support. N Engl J Med 1997;336:41-48.

4 Gosmanov AR, Umpierrez GE: Medical nutrition therapy in hospitalized patients with diabetes. Curr Diab Rep 2012;12:93-100.

5 Cave MC, Hurt RT, Frazier TH, Matheson PJ, Garrison RN, McClain CJ, et al: Obesity, inflammation, and the potential application of pharmaconutrition. Nutr Clin Pract 2008;23: 16-34.

6 van den Berghe G, Wouters P, Weekers F, Verwaest C, Bruyninckx F, Schetz M, et al: Intensive insulin therapy in the critically ill patients. N Engl J Med 2001;345:1359-1367.

7 Finfer S, Chittock DR, Su SY, Blair D, Foster $\mathrm{D}$, Dhingra $\mathrm{V}$, et al: Intensive versus conventional glucose control in critically ill patients. N Engl J Med 2009;360:1283-1297.

8 Klek S, Krznaric Z, Gundogdu RH, Chourdakis M, Kekstas G, Jakobson T, et al: Prevalence of malnutrition in various political, economic, and geographic settings. JPEN J Parenteral Enteral Nutr 2015;39:200-210.

9 Pleva M, Mirtallo JM, Steinberg SM: Hyperglycemic events in non-intensive care unit patients receiving parenteral nutrition. Nutr Clin Pract 2009;24:626-634.

10 Mesejo A, Montejo-Gonzalez JC, VaquerizoAlonso C, Lobo-Tamer G, Zabarte-Martinez M, Herrero-Meseguer JI, et al: Diabetes-specific enteral nutrition formula in hyperglycemic, mechanically ventilated, critically ill patients: a prospective, open-label, blind-randomized, multicenter study. Crit Care 2015; 19:390.

11 Jacobi J, Bircher N, Krinsley J, Agus M, Braithwaite SS, Deutschman C, et al: Guidelines for the use of an insulin infusion for the management of hyperglycemia in critically ill patients. Crit Care Med 2012;40:3251-3276.

12 American Diabetes Association: 13. Diabetes care in the hospital. Diabet Care 2016;39(suppl 1):S99-S104.

13 Cochrane Collaboration: Cochrane Handbook for Systematic Reviews of Interventions. 2011. http://www.cochrane-handbook.org/.

14 Liberati A, Altman DG, Tetzlaff J, Mulrow C, Gotzsche PC, Ioannidis JP, et al: The PRISMA statement for reporting systematic reviews and meta-analyses of studies that evaluate health care interventions: explanation and elaboration. J Clin Epidemiol 2009;62:e1-e34.

15 Higgins JP, Altman DG, Gotzsche PC, Juni P, Moher D, Oxman AD, et al: The Cochrane Collaboration's tool for assessing risk of bias in randomised trials. BMJ 2011;343:d5928.

16 Wells GA SB, O'Connell D, Peterson J, Welch V, Tugwell P: The Newcastle-Ottawa Scale (NOS) for Assessing the Quality of Nonrandomised Studies in Meta-Analyses. Ottawa Hospital Research Institute, 2000. http:// www.ohrica/programs/clinical_epidemiology/oxfordhtm.

17 Smith R, Baltagi S, Adelson PD, Smith C, Kochanek P, Clark R, et al: Understanding metabolic requirements of children after severe TBI. J Neurotrauma 2009;26:A99.

18 Balshem H, Helfand M, Schunemann HJ, Oxman AD, Kunz R, Brozek J, et al: GRADE guidelines: 3 . Rating the quality of evidence. J Clin Epidemiol 2011;64:401-406.

19 Chase JG, Shaw G, Le Compte A, Lonergan T, Willacy M, Wong XW, et al: Implementation and evaluation of the SPRINT protocol for tight glycaemic control in critically ill patients: a clinical practice change. Crit Care 2008; $12: R 49$

20 Dickerson RN, Wilson VC, Maish GO 3rd, Croce MA, Minard G, Brown RO: Transitional NPH insulin therapy for critically ill patients receiving continuous enteral nutrition and intravenous regular human insulin. JPEN J Parenteral Enteral Nutr 2013;37:506-516.

21 Murphy PM, Moore E, Flanagan DE: Glycaemic control in insulin requiring diabetes patients receiving exclusive enteral tube feeding in an acute hospital setting. Diab Res Clin Pract 2014;103:426-429.

22 Neff K, Donegan D, MacMahon J, O'Hanlon C, Keane N, Agha A, et al: Management of parenteral nutrition associated hyperglycaemia: a comparison of subcutaneous and intravenous insulin regimen. Ir Med J 2014;107: 141-143.

23 Holzinger U, Zauner A, Nimmerrichter P, Schiefermeier M, Ratheiser K, Zauner C: Metabolic inefficacy of a short-term low-dose insulin regimen in critically ill patients: a randomized, placebo-controlled trial. Wien Klin Wochenschr 2004;116:603-607.

24 Van Herpe T, Mesotten D, Wouters PJ, Herbots J, Voets E, Buyens J, et al: LOGIC-insulin algorithm-guided versus nurse-directed blood glucose control during critical illness the LOG-
IC-1 single-center, randomized, controlled clinical trial. Diab Care 2013;36:188-194.

25 Dortch MJ, Mowery NT, Ozdas A, Dossett L, Cao H, Collier B, et al: A computerized insulin infusion titration protocol improves glucose control with less hypoglycemia compared to a manual titration protocol in a trauma intensive care unit. J Parenteral Enteral Nutr 2008; 32:18-27.

26 Oghazian MB, Javadi MR, Radfar M, Torkamandi H, Sadeghi M, Hayatshahi A, et al: Effectiveness of regular versus glargine insulin in stable critical care patients receiving parenteral nutrition: a randomized controlled trial. Pharmacotherapy 2015;35:148-157.

27 Dickerson RN, Lynch AM, Maish GO 3rd, Croce MA, Minard G, Brown RO: Improved safety with intravenous insulin therapy for critically ill patients with renal failure. Nutrition 2014;30:557-562.

28 Hsia E, Seggelke SA, Gibbs J, Rasouli N, Draznin B: Comparison of 70/30 biphasic insulin with glargine/lispro regimen in noncritically ill diabetic patients on continuous enteral nutrition therapy. Nutr Clin Pract 2011;26:714-717.

29 Korytkowski MT, Salata RJ, Koerbel GL, Selzer F, Karslioglu E, Idriss AM, et al: Insulin therapy and glycemic control in hospitalized patients with diabetes during enteral nutrition therapy: a randomized controlled clinical trial. Diab Care 2009;32:594-596.

30 Leelarathna L, English SW, Thabit H, Caldwell K, Allen JM, Kumareswaran K, et al: Feasibility of fully automated closed-loop glucose control using continuous subcutaneous glucose measurements in critical illness: a randomized controlled trial. Criti Care 2013; 17:R159.

31 Grainger A, Eiden K, Kemper J, Reeds D: A pilot study to evaluate the effectiveness of glargine and multiple injections of lispro in patients with type 2 diabetes receiving tube feedings in a cardiovascular intensive care unit. Nutr Clin Pract 2007;22:545-552.

32 Cook A, Burkitt D, McDonald L, Sublett L: Evaluation of glycemic control using NPH insulin sliding scale versus insulin aspart sliding scale in continuously tube-fed patients. Nutr Clin Prac 2009;24:718-722.

33 Hakeam HA, Mulia HA, Azzam A, Amin T: Glargine insulin use versus continuous regular insulin in surgical noncritically ill patients receiving parenteral nutrition: randomized controlled study. JPEN J Parenteral Enteral Nutr 2016, Epub ahead of print. 
34 Jakoby MG, Nannapaneni N: An insulin protocol for management of hyperglycemia in patients receiving parenteral nutrition is superior to ad hoc management. JPEN J Parenteral Enteral Nutr 2012;36:183-188.

35 Thomas AN, Marchant AE, Ogden MC, Collin S: Implementation of a tight glycaemic control protocol using a web-based insulin dose calculator. Anaesthesia 2005;60:10931100 .
36 Van den Berghe G: Beyond diabetes: saving lives with insulin in the ICU. Int J Obesity Related Metab Dis 2002;26(suppl 3):S3-S8.

37 NICE-SUGAR Study Investigators, Finfer S, Liu B, Chittock DR, Norton R, Myburgh JA, et al: Hypoglycemia and risk of death in critically ill patients. N Engl J Med 2012;367: 1108-1118.
38 Lansang MC, Umpierrez GE: Inpatient hyperglycemia management: a practical review for primary medical and surgical teams. Cleve Clin J Med 2016;83:S34-S43.

39 Chase JG, Shaw GM, Wong XW, Lotz T, Lin J, Hann CE: Model-based glycaemic control in critical care: a review of the state of the possible. Biomed Signal Process Control 2006;1: 3-21.

40 Koretz RL, Lipman TO: Understanding systematic reviews and meta-analyses. JPEN J Parenteral Enteral Nutr 2016;41:316-323. 